\title{
CYTOGENETIC ANALYSIS OF PATIENTS WITH AMBIGUOUS GENITALIA
}

\author{
Sara Ali Zaidi, Asad Mahmood, Rafia Mahmood, Aamna Latif, Helen Mary Robert, Sarah Fatimah
}

Armed Forces Institute of Pathology/National University of Medical Sciences (NUMS) Rawalpindi Pakistan

\section{ABSTRACT}

Objective: To determine the magnitude and classification of cases of ambiguous genitalia presenting to our setup. Study Design: Cross-sectional study.

Place and Duration of Study: Department of Haematology, Armed Forces Institute of Pathology, Rawalpindi Pakistan, from Aug 2018 to Feb 2019.

Methodology: All the patients with ambiguous genitalia referred for cytogenetic analysis, were included in the study. The patients were subjected to a detailed history and physical examination. The record of radiological investigations was were obtained. Cytogenetic analysis was performed using the conventional G-banding technique. Hormonal testing included 17hydroxyprogesterone (17-OHP) levels was also performed.

Results: Fifty-one cases of ambiguous genitalia were studied. The median age was 15 months. Thirty-three patients (64.7\%) had a 46XY karyotype, $17(33.3 \%)$ had a 46XX karyotype while $1(1.9 \%)$ had 45X/46, XY mosaic karyotype. Thirty patients $(58.8 \%)$ were products of consanguineous marriage. Congenital adrenal hyperplasia was diagnosed in 12 cases (70.5\%) of 46 XX karyotype and in 3 cases $(9 \%)$ of 46XY karyotype.

Conclusion: Ambiguous genitalia, currently categorized as disorders of sex development, are not uncommon in our populartion. Increased awareness and early diagnosis are crucial to prevent life threatening complications of congenital adrenal hyperplasia, to determine sex of rearing, and to counsel the parents or patients.

Keywords: Ambiguous genitalia, Congenital adrenal hyperplasia, Cytogenetic analysis, Disorders of sex development.

How to Cite This Article: Zaidi SA, Mahmood A, Mahmood R, Latif A, Robert HM, Fatimah S. Cytogenetic Analysis of Patients with Ambiguous Genitalia . Pak Armed Forces Med J 2021; 71(6): 2161-2165. Doi: https://doi.org/10.51253/pafmj.v71i6.5007

This is an Open Access article distributed under the terms of the Creative Commons Attribution License (https://creativecommons.org/licenses/by-nc/4.0/), which permits unrestricted use, distribution, and reproduction in any medium, provided the original work is properly cited.

\section{INTRODUCTION}

Disorders of sex development (DSD) are a group of congenital conditions characterized by atypical development of chromosomal, gonadal or phenotypic sex. Previously these were termed as "Intersex disorders". ${ }^{1}$ The most common clinical presentation is variable degree of genital ambiguity in the neonatal period. However, if genital ambiguity is not present or remains unnoticed, they can present later in life with primary amenorrhea, inguinal hernia or virilization in an apparent female, gynaecomastia or delayed puberty in an apparent male, or infertility in both genders. ${ }^{2}$ Genital ambiguities are reported to occur at an incidence of 1 per 4500 live births. ${ }^{1}$

DSD arise from abnormalities in gonadal determination or sex differentiation, which are under complex control of various genes and hormones. The chromosomal sex (XX or XY) determines the gonadal sex, which is translated into the phenotypic sex. Under the influence of the sex determining region of the $Y$ chromosome (SRY gene), the undifferentiated, bipotential gonad develops into the testis. Under the action of hor-

Correspondence: Dr Sara Ali Zaidi, Department of Haematology, Armed Forces Institute of Pathology, Rawalpindi Pakistan

Received: 14 Aug 2020; revision received: 22 Dec 2020; accepted: 29 Dec 2020 mones secreted from testes, the internal ducts and external genitalia differentiate into a male sex phenotype. In the absence of the SRY gene, female sexual differentiation occurs. The bipotential gonad develops into the ovary, and the internal and external genitalia differentiate into that of a female in absence of testicular hormones. ${ }^{3}$ Downstream of the SRY gene, other genes and transcription factors have important roles in testes development. ${ }^{4}$ The ovarian development also depends on expression of critical genes. Any error during sex determination or differentiation, results in ambiguous genitalia or DSD. 5

The birth of a child with ambiguous genitalia is a medical and social emergency. It is not only a cause of emotional stress for the family but also a diagnostic challenge for the physician. ${ }^{6}$ Urgent evaluation is necessary to establish a correct diagnosis as soon as possible. The aim should be, to counsel the parents about the treatment options and gender assignment in time to prevent medical and psychological complications. DSD management requires a multi-disciplinary team that should include a pediatric endocrinologist, pediatric urologist, geneticist, and a psychologist. ${ }^{7}$

Patients should be evaluated by detailed family and antenatal history, thorough physical examination, 
karyotyping, radiological imaging, and hormonal testing. Molecular testing for various gene mutations, where available, should also be performed. Patients should be investigated in a tertiary care setup where all these diagnostic facilities are available. The 2006 consensus statement of the ESPE proposed a revised nomenclature and classification of DSD taking into account genetic makeup and descriptive terminologies. The main categories include 46XX DSD, 46XY DSD, and DSD with sex chromosome abnormalities. ${ }^{1}$ Cytogenetic analysis to establish the karyotype is thus essential for classification and diagnosis of DSD cases. As the differential diagnosis and further investigations depend on the karyotype, it is the first-line testing in DSDs. It is also essential for excluding mosaicism or other chromosomal abnormalities. ${ }^{8}$

Armed Forces Institute of Pathology (AFIP) is a tertiary care referral centre providing advanced diagnostic facilities like cytogenetic analysis and hormonal testing. Currently there is limited data available in Pakistan about DSD cases. This study was conducted with an aim to determine the frequency of DSD cases presenting to our setup and to classify them according to their chromosomal makeup.

\section{METHODOLOGY}

This cross-sectional study was conducted at Haematology Department, Armed Forces Institute of Pathology (AFIP), Rawalpindi, from August 2018 to March 2019.

Inclusion Criteria: All the patients referred for cytogenetic analysis with the indication of ambiguous genitalia were included in the study.

Exclusion Criteria: Patients referred for cytogenetic analysis due to other indications, e.g. primary amenorrhoea in females or pubertal delay in males, were excluded.

Sample size was calculated using WHO sample size calculator. Study was conducted after the ethical approval of the institutional review board (IRB no. 18/859). All the patients were included in this study after the informed consent of patients or their parents. Non-probability consecutive sampling technique was used.

All the cases were subjected to a detailed history which included age of presentation, sex of rearing, consanguinity, family history, history of sibling death, maternal exposure to androgens during pregnancy or virilization symptoms in the mother. Criteria for ambiguous genitalia included overt genital ambiguity, apparent female genitalia with enlarged clitoris, posterior labial fusion, or inguinal/labial mass, apparent male genitalia with bilateral undescended testes, hypospadias, or micropenis. Physical examination included, genital examination with stress on presence or absence of testes and their site, and the presence or absence of secondary sexual characteristics in older patients. Patients were also examined for dysmorphic features or other congenital anomalies. Record of radiological investigations was obtained from the patients. These included abdomino-pelvic ultrasonography and/or MRI.

Cytogenetic analysis included chromosomal karyotyping using the conventional G-banding technique. $3 \mathrm{ml}$ of heparinized peripheral blood samples were collected. Samples were subjected to 72 hours culture in RPMI 1640 medium at 37-C. Harvesting was done to obtain metaphases by first adding $1 \%$ colchicine followed by incubation, centrifugation and addition of hypotonic solution of $1 \% \mathrm{KCl}$. Fixation was done by methanol and glacial acetic acid in a ratio of 3:1 after which slides were prepared. Slides were examined under the microscope after Leishman staining to look for presence of at least 20 metaphases that rendered the culture successful. After Giemsa trypsin banding slides were analyzed by Cytovision semi-automated image analysis and capture system. A minimum of 20 metaphases were analyzed and interpreted according to the international system of cytogenetic nomenclature (ISCN).

The results of karyotyping were used to classify patients as having 46XX DSD, 46XY DSD or Sex chromosome DSD. All patients were also tested for 17-hydroxyprogesterone (17-OHP) levels to exclude Congenital Adrenal Hyperplasia (CAH), as advised by their referring physicians. All the information was entered on a predesigned pro forma. The data was analyzed by SPSS version 24. Quantitative variables were presented in terms of median while qualitative variables as frequency and percentages.

\section{RESULTS}

Fifty one cases of ambiguous genitalia were studied. Their age ranged from 3 days - 30 years with a median age of 15 months. Among them, 29 (56.8\%) were in the age group of $0-1$ years, $13(25.4 \%)$ were in the age group of 2-6 years while $2(3.9 \%)$ were aged 711 years. Four $(7.8 \%)$ belonged to the age group of $12-16$ years and $3(5.8 \%)$ were between $17-30$ years as shown in Figure-1. Thirty-three (64.7\%) patients had a 46XY karyotype, $17(33.3 \%)$ had a 46XX karyotype while $1(1.9 \%)$ had a $45 \mathrm{X} / 46, \mathrm{XY}$ mosaic karyotype. 
Thus they were classified as having 46XY DSD, 46XX DSD and sex chromosome DSD respectively shown in Figure-2.

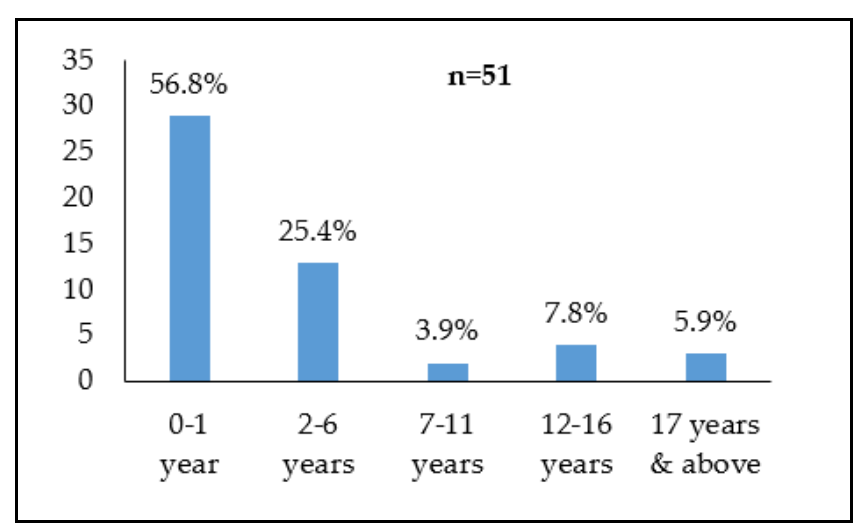

Figure-1: Age distribution of study population.

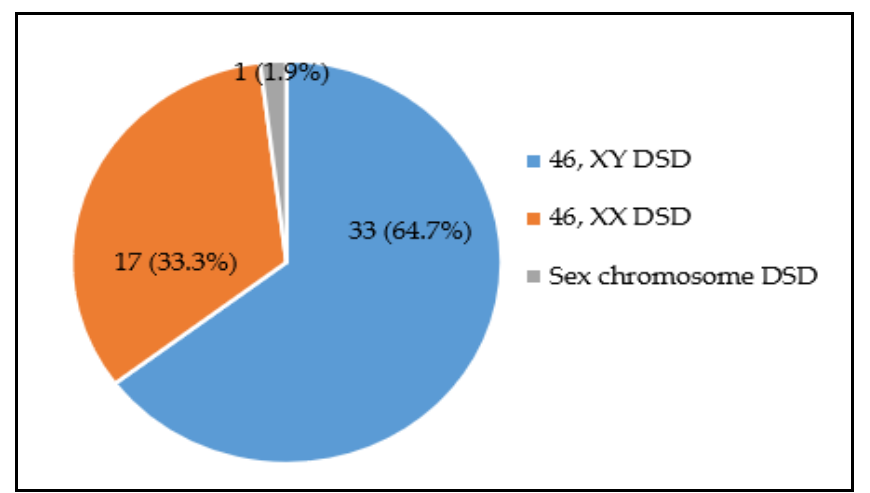

Figure-2: Classification of cases of ambiguous genitalia in this series.

Out of 51 patients, $30(58.8 \%)$ were products of consanguineous marriage. The consanguinity rate was $20(60.6 \%)$ among 46XY DSD and $10(58.8 \%)$ among 46XX DSD cases (Table). Family history of ambig-uous genitalia and/or congenital anomalies was found in 8 cases $(15.6 \%)$.

Table: Consanguinity rate among disorders of sex development (DSD) cases.

\begin{tabular}{l|c|c}
\hline Classification & No. of Cases & Consanguinity \\
\hline 46XY DSD & 33 & $20(60.6 \%)$ \\
\hline 46XX DSD & 17 & $10(58.8 \%)$ \\
\hline Sex chromosome DSD & 1 & - \\
\hline Total & 51 & $30(58.8 \%)$ \\
\hline
\end{tabular}

Among 46XY DSD cases ( $\mathrm{n}=33), 27(81.8 \%)$ were being reared as males while $6(18.2 \%)$ were being reared as females. Among 46XX DSD cases $(n=17), 9(53 \%)$ were being raised as females while $8(47 \%)$ were being raised as males. In the 6 cases with 46XY karyotype being raised as females and the 8 cases of 46XX karyotype being raised as males, karyotyping was repeated to confirm the diagnosis. One case of $45 \mathrm{X} / 46, \mathrm{XY}$ mosaic karyotype was being raised as a female. Its karyotype was also confirmed by repeat testing and examination of 40 metaphases.

Congenital Adrenal Hyperplasia (CAH) was diagnosed in 12 cases $(70.5 \%)$ of 46XX DSD and in 3 cases $(9 \%)$ of $46 \mathrm{XY}$ DSD based on raised 17-OHP levels. On imaging studies, all patients with 46XX karyotype had internal female genitalia except for $3(17.6 \%)$, who had bilateral undescended testes and no internal female genitalia. No patients with 46XY karyotype had any internal female genitalia. $9(27.7 \%)$ had testes located within scrotal sacs while $13(39.4 \%)$ had bilateral or unilateral undescended testes; both of these groups had normal male internal genitalia. $6(18.2 \%)$ cases had rudimentary testes while $5(15.1 \%)$ cases had absent testes; both of these groups did not have any male internal genitalia. One case of 45X/46,XY karyotype (Turner Mosaic) had female-like ambiguous genitalia with bilateral inguinal testes, however ultrasound revealed an infantile uterus with absent ovaries.

\section{DISCUSSION}

There is scarce data available on the prevalence and etiologies of ambiguous genitalia/DSD in Pakistan. The process of human sexual development is tightly regulated by various genes and hormones. Any disruption during this process results in disorders of sexual development that have variable presentations and multiple etiologies. They can present at birth as ambiguous external genitalia or may manifest later in life. A newborn with malformed genitalia is a diagnostic and therapeutic challenge for the treating pediatrician. A multidisciplinary team should work closely with the family to establish the correct diagnosis of the abnormality and assign the gender based on the karyotype, endocrine function, fertility potential and surgical options. ${ }^{9}$

In the present study, majority of the patients of ambiguous genitalia presented in infancy 29 (56.8\%). This is consistent with published data. A study conducted by Amolo et a ${ }^{10}$ showed that $58 \%$ of the cases were less than 1 year of age.

Cytogenetic analysis revealed that the most common karyotype was 46XY $33(64.7 \%)$. Mazen et al. ${ }^{11}$ and Al-Agha et al, ${ }^{12}$ have also observed a higher incidence of 46XY DSD in their studies, $65.9 \%$ and $56.8 \%$, respectively. However, a study conducted by Al-Jurayyan, 13 showed that $46 \mathrm{XX}$ DSD was more common than $46 \mathrm{XY}$ DSD $(65.4 \%$ and $34.6 \%$, respectively). 
Review of published data shows that majority of DSD cases have a normal karyotype i.e. 46XY or 46XX, while karyotype abnormalities leading to sex chromosome DSD are less common. ${ }^{14,15}$ Our study also showed similar results with only 1 case $(1.9 \%)$ with a 45 , X/46,XY mosaic karyotype.

$\mathrm{CAH}$ was diagnosed in 12 cases $(70.5 \%)$ of $46 \mathrm{XX}$ DSD and 3 cases (9\%) of 46XY DSD based on raised 17-OHP levels. CAH is the most common cause of DSD worldwide, with an estimated incidence of 1:14001:1500 live births; however, it varies among different ethnic groups. ${ }^{16}$

In the present study, $58.8 \%$ of the cases were products of consanguineous marriages. A study from Sudan ${ }^{17}$ observed a consanguinity rate of $70 \%$. Mazen et al, ${ }^{11}$ have observed a consanguinity rate of $62.8 \%$ among Egyptian patients. Bashamboo and McElreavey ${ }^{18}$ have suggested that high consanguinity rates may have a role in the higher incidence of DSD cases among certain populations. The incidence of ambiguous genitalia in Arab countries is higher than the incidence reported in studies from European countries. ${ }^{19,20}$ Arab, African and Asian countries have higher incidence of consanguineous marriages. Consanguinity leads to the accumulation of abnormal recessive genes, and thus results in an increased incidence of conditions manifested by autosomal recessive inheritance. $\mathrm{CAH}$ has an autosomal recessive pattern of inheritance, thus leading to its increased incidence in the endogamous communities. ${ }^{21}$ Few rare conditions of 46XY DSD also have an autosomal recessive inheritance.

Our study showed that a relatively high number of cases of ambiguous genitalia reported to our setup in a short period of time (51 cases in 6 months) as compared to the studies done by Al-Mutair et al. ${ }^{22}$ (120 patients in 10 years), and Praburam 23 (165 patients in 18 years). This reflects the high burden of DSD in our population. In addition, the frequency is higher than reported by Manzoor et al. ${ }^{24}$ (300 patients in 7 years) and Raza et al, ${ }^{25}$ (429 patients in 5 years) in other parts of Pakistan. This may be due to the reason that our cytogenetic lab caters to heavy referrals from all over the country.

Karyotype analysis has a central role in the investigation of DSD. It is essential to classify DSD and helps in narrowing the differential diagnosis and planning subsequent hormonal investigations. Results of karyotyping, radiological imaging and biochemical assays suggest the etiological diagnosis. Early diagnosis is crucial to prevent life threatening complications in case of $\mathrm{CAH}$, to determine the most appropriate sex of rearing, to plan hormonal and/or surgical management, and to counsel the parents and patients.

\section{CONCLUSION}

The results of our study indicate that these conditions are not uncommon in our population. Further studies to determine the actual prevalence and various etiologies of DSD cases in Pakistan are required. This will help in creating diagnostic algorithms and management guidelines.

\section{Conflict of Interest: None.}

\section{Authors' Contribution}

SAZ: Conception and design of study, collection and analysis of data and drafting of manuscript, AM: Review of manuscript, final approval, RM: Data analysis, Interpretation of data, literature review, AL: Data collection, HMR: Literature review, SF: Manuscript preparation.

\section{REFERENCES}

1. Hughes IA, Houk C, Ahmed SF, Lee PA. LWPES Consensus group; ESPE consensus group. Consensus statement on management of intersex disorders. Arch Dis Child 2006; 91(7): 554-563.

2. Ahmed SF, Achermann JC, Arlt W, Balen AH, Conway G, Edwards ZL, et al. UK guidance on the initial evaluation of an infant or an adolescent with a suspected disorder of sex development. Clin Endocrinol (Oxf) 2011; 75(1): 12-26.

3. Fisher AD, Ristori J, Fanni E, Castellini G, Forti G, Maggi M. Gender identity, gender assignment and reassignment in individuals with disorders of sex development: a major of dilemma. J Endocrinol Invest 2016; 39(11): 1207-1224.

4. Bashamboo A, McElreavey K. Human sex-determination and disorders of sex-development (DSD). Semin Cell Dev Biol 2015; 45(1): 77-83.

5. Croft B, Ayers K, Sinclair A, Ohnesorg T. Review disorders of sex development: the evolving role of genomics in diagnosis and gene discovery. Birth Defects Res C Embryo Today 2016; 108(4): 337-350.

6. McCann-Crosby B, Sutton VR. Disorders of sexual development. Clin Perinatol 2015; 42(2): 395-412.

7. Cools M, Nordenström A, Robeva R, Hall J, Westerveld P, Flück $\mathrm{C}$, et al. Caring for individuals with a difference of sex development (DSD): a consensus statement. Nat Rev Endocrinol 2018; 14(7): 415-429.

8. Ogilvy-Stuart AL, Brain CE. Early assessment of ambiguous genitalia. Arch Dis Child 2004; 89(5): 401-407.

9. Hiort O, Birnbaum W, Marshall L, Wünsch L, Werner R, Schröder T, et al. Management of disorders of sex development. Nat Rev Endocrinol 2014; 10(9): 520.

10. Amolo P, Laigong P, Omar A, Drop S. Etiology and clinical presentation of disorders of sex development in kenyan children and adolescents. Int J Endocrinol 2019, [Internet] Available at : https://www.hindawi.com/journals/ije/2019/2985347/ (Accessed on October 20, 2019)

11. Mazen I, Hiort O, Bassiouny R, El Gammal M. Differential diagnosis of disorders of sex development in Egypt. Horm Res 2008; 70(2): 118-123.

12. Al-Agha AE, Thomsett MJ, Batch JA. The child of uncertain sex: 17 years of experience. J Paediatr Child Health 2001; 37(4): 348351. 


\section{Ambiguous Genitalia}

13. Al-Jurayyan NA. Ambiguous genitalia: two decades of experience. Ann Saudi Med 2011;31(3): 284-288.

14. Kumari VA, Vani A. Ambiguous genitalia: a clinical and chromosomal study. Int J Res Med Sci 2017; 3(12): 3743-3748.

15. Thillainathan S, Sirisena ND, Kariyawasam KW, Jayasekara RW. Cytogenetic analysis of chromosomal abnormalities in Sri Lankan children. World J Pediatr 2015; 11(4): 374-379.

16. Lee PA, Nordenström A, Houk CP, Ahmed SF, Auchus R. Global disorders of sex development update since 2006: perceptions, approach and care. Horm Res Paediatr 2016; 85(3): 158-180.

17. Abdullah MA, Saeed U, Abass A, Lubna K, Weam A, Ali AS, et al. Disorders of sex development among Sudanese children: 5-year experience of a pediatric endocrinology clinic. J Pediatr Endocrinol Metab 2012; 25(11-12): 1065-1072.

18. Bashamboo A, McElreavey K. Consanguinity and disorders of sex development. Hum Hered 2014; 77(1-4): 108-117.

19. Abdullah MA, Katugampola M, Al-Habib S, Al-Jurayyan N, AlSamarrai A, Al-Nuaim A, et al. Ambiguous genitalia: medical, socio-cultural and religious factors affecting management in Saudi Arabia. Ann Trop Paediatr 1991; 11(4): 343-348.
20. Thyen U, Lanz K, Holterhus PM, Hiort O. Epidemiology and initial management of ambiguous genitalia at birth in Germany. Horm Res 2006; 66(4): 195-203.

21. Alshabab LI, Alebrahem A, Kaddoura A, Al-Fahoum S. Congenital adrenal hyperplasia due to 21-hydroxylase deficiency: a five-year retrospective study in the Children's Hospital of Damascus, Syria. Qatar Med J 2015; 2015(1): 11-15.

22. Al-Mutair A, Iqbal MA, Sakati N, Ashwal A. Cytogenetics and etiology of ambiguous genitalia in 120 pediatric patients. Ann Saudi Med 2004; 24(5): 368-372.

23. Praburam PM. Medical audit of children with ambiguous genitalia-review of children treated over 18 years. Int J Med Res Health Sci 2015; 4(4): 879-883.

24. Manzoor J, Aftab S, Yaqoob M. Ambiguous genitalia: An overview of 7 years experience at the Children's Hospital \& Institute of Child Health, Lahore, Pakistan. Pak J Med Sci 2019; 35(1): 151155.

25. Raza J, Mazen I. Achieving diagnostic certainty in resourcelimited settings. Endocr Dev 2014; 27(1): 257-267. 